\title{
VERIFICATION OF ${ }^{235} U,{ }^{238} U$ BY MATHEMATICAL CALIBRATION CURVE FOR POINT SOURCES BY USING MONTE CARLO
}

\section{S. A. Makhlouf}

Faculty of Science, Al-Azhar University, Assiut, Egypt.

\begin{abstract}
: detector distances (D) by using Monte Carlo [MC] simulation method.

\section{INTRODUCTION}

In order to fulfill its national and international safeguards commitments, a state should establish and maintain a state system of accounting for and control (SSAC) of nuclear materials (NMs) [1]. The main function of that system is to verify NM in such state. Verification activities are achieved via two main steps,
\end{abstract}

The calibration of $\gamma$-ray spectrometer was performed by means of a set of certified standard point sources of energies that cover the energy range of interest. The value of the absolute full energy peak efficiency "Eab " could be found by measuring "CR" experimentally and the use of the known value of "A ". Also, it is possible to calculate "Eab" for a certain source to

The obtained results indicated that the fitting is identical to the experimental curve and MCNP calculations, that is both these two methods (experimental and MCNP) can be used to extend the curve to cover the high energy range. It is possible to drop any line energy inside the used energy range to estimate the absolute full energy peak efficiency, (for example, the energies 185.7, 1001 and $1275 \mathrm{keV}$ corresponding to the sources 235U, 238U and 22Na). However, it can be estimated by using any method from the previous methods (experimental or MCNP in addition to the fitting equation).

Keywords: point sources, experimental, fitting equation, MCNP, A mathematical calibration curve.

First: The facility operators are obliged to provide the inspector with all information relevant to NM inventory and inventory changes. Then, the NM quantities received, produced, shipped, lost or otherwise removed from inventory, as well as quantities basically present in the inventory are balanced within a certain limited area using different measuring techniques and accounting procedures. It is also required to evaluate the precision and accuracy of the measurements and estimate the overall uncertainty [2].

Second: the measured NM quantities are compared with those declared by the facility operators. The acceptance of operators' declarations (operator-inspector differences) depends on some criteria, which are related to the accuracy and precision of the measurement results for both inspectors and operators [3, 4].

Nuclear fuel used in nuclear reactors (research or power reactors) may be manufactured from Low-Enriched Uranium (LEU) oxide pow- ders in the form of pellets, rods, plates, ... etc., and assembled as fuel assemblies, elements or bundles. Standards of NMs of well-known enrichments and of similar characteristics to the assayed fuel sample are usually needed for performing enrichment calibration of the measuring system. Such NM standards may not be available or suitable for the purpose in different situations. Recent studies are directed towards overcoming this problem $[5,6]$.

Monte Carlo (MC) simulation technique, which is becoming progressively popular [7-9], has been used by many authors, many years ago, to simulate the process of gamma-ray detection. It was used to calculate the response characteristics of different germanium detectors at monoenergetic and different gamma-ray energy ranges [10-18]. It was also used for detectors calibration, either directly or through combination with experimental measurements [7,8]. Relative efficiency curves determination [19] and simulation of energy spectra [20] were also performed. General MC codes such as MCNP, GEANT and EGS4, are being extensively tested for such calculations [21].

\section{MATHEMATICAL CALIBRATION CURVE FOR POINT SOURCES}

A mathematical calibration curve can be drown for any apparatus using MC (the general-purpose Monte Carlo Neutron and Photon 
(transport code) (MCNP)). It depends on different parameters of the used sources such as size, energy and "source-detector" distance.

In this work, a mathematical calibration curve will be constructed for some point sources at different energy lines.

The basic definition of the absolute full energy peak (FEP) photon detection efficiency $\left(\varepsilon_{a b}\right)$ is,

Total number of the detected photons under the FEP $\varepsilon_{a b}=$

Total number of the photons emitted by the source at the same energy

For a geometrical configuration of a $\gamma$-emitting source located at a distance "D" in front of a Hyper Pure Germanium (HPGe) detector along its central line, the net counte rate " $\mathrm{C}_{\mathrm{R}}$ " measured by the detector measuring system at a specified full energy $\gamma$-ray peak due to a source can be expressed to be [22]:

$$
\mathrm{C}_{\mathrm{R}}=A \cdot \Omega_{f} \cdot \mathrm{A}_{\mathrm{t}} \cdot \varepsilon_{i} \cdot \mathrm{F} \text {, }
$$

Where $A$ is the total activity of $\gamma$-ray emitted from the source in all directions (assumed isotropic) for a point sources, $\Omega_{f}$ is the fractional solid angle of the source subtended by the detector, $\mathrm{A}_{t}$ is the total attenuation correction factor for the " $D$ " setup, $\varepsilon_{i}$ is the full energy peak intrinsic efficiency of the detector at a specified $\gamma$-energy and $\mathrm{F}$ is the correction factor related to the electronic losses, background and coincidence summing.

In the optimum situation of the measuring system, where the effects are due to the electronic losses, the background and coincidence summing could be minimized and the factor $\mathrm{F}$ could be ignored.

The value of " $\Omega_{f} \mathrm{~A}_{\mathrm{t}} \cdot \varepsilon_{i}$ " represents the absolute full energy peak efficiency of the detector measuring system " $\varepsilon_{a b}$ ", i.e,

$$
\varepsilon_{a b}=\Omega_{f} \cdot \mathrm{A}_{\mathrm{t}} \cdot \varepsilon_{i}
$$

Then, Eq. (2) may be put in the form

$$
\mathrm{C}_{\mathrm{R}}=\text { A. } \varepsilon_{a b} \text {. }
$$

\section{COUNT RATE MEASUREMENT}

Count rates due to ${ }^{235} \mathrm{U}$ were measured using HPGe detector. All sources were measured with the same configurations with respect to the de- tector. Each source was located so that the axis of symmetry of the detector passes through the central point of the measured source and the sample-to-detector distance was chosen such that counting losses due to electronics were minimized. The detector dead time did not exceed $1 \%$. Three runs were taken for each measured sample. Thus the mean values of the measured count rates were used in calculations. The fullenergy peak areas are determined by manual selection. All measurements were performed applying non-destructive assay (NDA) technique at the safeguard laboratories of the National Center for Nuclear Safety and Radiation Control. This location belongs to the Egyptian Atomic Energy Authority and subjected to the safeguards agreement between Egypt and the International Atomic energy Agency (IAEA).

\section{MCNP}

\subsection{The Monte Carlo Method}

MCNP is a general-purpose, continuous-energy, generalized-geometry, time-dependent, coupled neutron/photon/electron Monte Carlo transport code. It can be used in several transport modes: neutron only, photon only, electron only, combined neutron/photon transport (where the photons are produced by neutron interactions), neutron/photon/electron, photon/electron, or electron/photon. The neutron energy regime is from $10^{-11} \mathrm{MeV}$ to $20 \mathrm{MeV}$, and the photon and electron energy regime are from $1 \mathrm{keV}$ to 1000 $\mathrm{MeV}$. The capability to calculate $\mathrm{k}_{\text {eff }}$ eigen values for fissile systems is also available.

\subsection{The Monte Carlo Method Theory}

Monte Carlo method can be used to duplicate theoretically a statistical process (such as the interaction of nuclear particles with materials) and is particularly useful for complex problems that cannot be modeled by computer codes that use deterministic methods. The individual probabilistic events that comprise a process are simulated sequentially. The probability distributions governing these events are statistically sampled to describe the total phenomenon. The statistical sampling process is based on the selection of random numbers analogous to throw dice in a gambling casino hence the name "Monte Carlo." 
Verification of ${ }^{235} \mathrm{U},{ }^{238} \mathrm{U}$ by Mathematical calibration curve

In particle transport, Monte Carlo technique is pre-eminently realistic (a theoretical experiment). It consists of actually following each of many particles from a source throughout its life to its death in some terminal category (absorption, escape, etc.). Probability distributions are randomly sampled using transport data to determine the outcome of each step of its life

\section{RESULTS AND DISCUSSION OF POINT SOURCES AT D: 40 CM}

The calibration of $\gamma$-ray spectrometer was performed by means of a set of certified standard point sources of emission energies that cover the energy range of interest. The value of " $\varepsilon_{a b}$ " could be found from Eq. (4) by measuring " $\mathrm{C}_{R}$ " experimentally and the use of the known value of " $A$ ". Also, it is possible to calculate " $\varepsilon_{a b}$ " for a certain $\mathrm{D}$ configuration by using $\mathrm{MC}$ simulation method.

The absolute full energy peak efficiency " $\varepsilon_{a b}$ " of the HPGe detector system was investigated with a set of point sources [Table (1)]. The geometrical configuration was arranged with $\mathrm{D}$ distance fixed at $40 \mathrm{~cm}$.

The experimental " $\varepsilon_{a b}$ " at D equals $40 \mathrm{~cm}$ for point sources as a function of energy with the MCNP calculated values of $\varepsilon_{a b}$ for the same configuration is presented in Fig. (1).

From Figure (1) it is notable that the fitting is identical for the experimental curve and MCNP calculation. However, the curve can be extended to cover the high energy range. So, in order to be sure that the plotted curve may be valid for higher energy range, the line energy of

Table (1). Gamma radiation from some point sources*.

\begin{tabular}{|c|c|r|c|c|c|r|r|r|}
\hline Source & Co-57 & $\begin{array}{r}\text { Co- } \\
57\end{array}$ & $\begin{array}{r}\text { U- } \\
235\end{array}$ & Cs-137 & Mn-54 & $\begin{array}{r}\text { Co- } \\
60\end{array}$ & $\begin{array}{r}\text { Na- } \\
22\end{array}$ & $\begin{array}{r}\text { Co- } \\
60\end{array}$ \\
\hline $\begin{array}{c}\text { Energyline } \\
((\mathrm{keV}\end{array}$ & 122.1 & 136.5 & 185.7 & 661.7 & 834.8 & 1173 & 1275 & 1333 \\
\hline $\begin{array}{c}\text { Activity } \\
(\mu \mathrm{Ci})\end{array}$ & 10.57 & 10.57 & 0.198 & 9.862 & 10.27 & 8.637 & 9.826 & 8.637 \\
\hline
\end{tabular}

* Certified Standard point sources [[23]; with total uncertainty $\pm 3.0 \%]$.

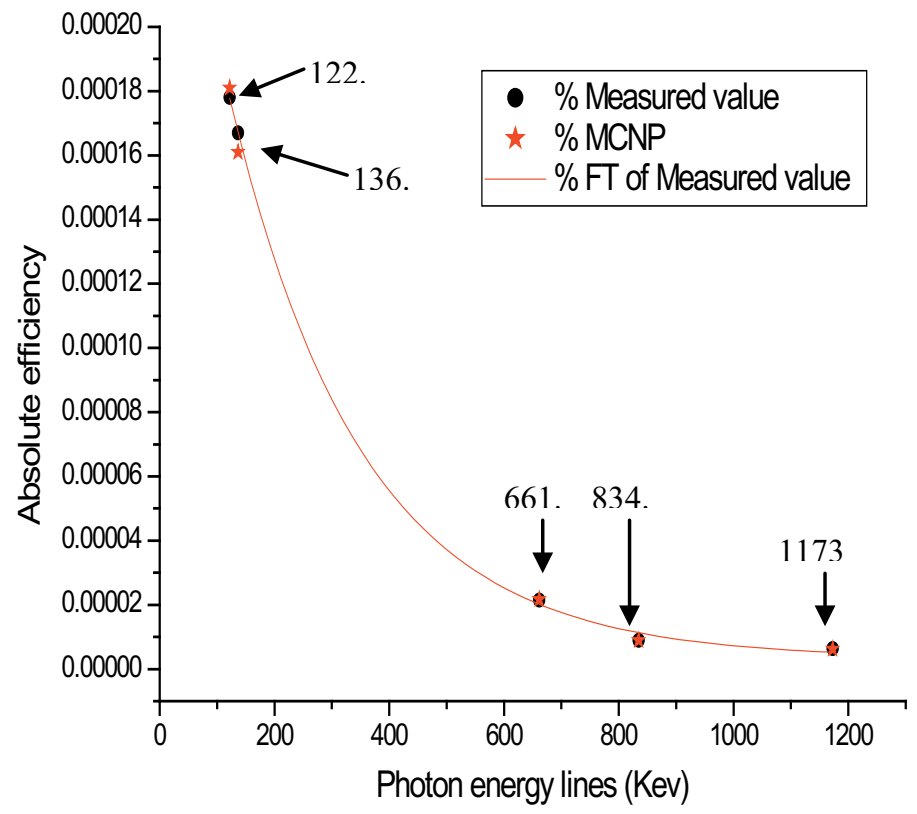

Figure (1). The absolute full energy peak efficiency calculated by MCNP, fitting equation and experimental absolute full energy peak efficiency versus the $\gamma$-energy lines for point sources at $D$ equals $40 \mathrm{~cm}$.

[FE has the form: $\left.y=A_{1} e^{*}\left(-x / t_{1}\right)+A_{2} e^{*}\left(-x / t_{2}\right)+A_{3} e^{*}\left(-x / t_{3}\right)+y_{0}\right]$

$\left[\mathrm{y}_{0}=0, \quad \mathrm{~A}_{1}=\mathbf{0 . 0 0 0 1 5 6 0 1 3}, \quad \mathrm{t}_{1}=\mathbf{2 2 3 . 9 1 0 2 2 ,} \quad \mathrm{A}_{2}=\mathbf{0 . 0 0 0 1 4 0 4 2 5 ,} \quad \mathrm{t}_{2}=\mathbf{2 3 7 . 6 1 9 6 3 ,} \quad \mathrm{A}_{3}=\right.$ $\left.0.00000337062, \quad t_{3}=-8.85449 E 122\right]$

$\mathbf{y}=\varepsilon_{a b}, \quad \mathbf{X}=$ energy line,$\quad \mathbf{F E}=$ Fitting Equation. 
(Co-60, $1333 \mathrm{keV}$ ) was measured and calculated by using MCNP. The results based on both the experimental and MCNP are identical as shown in Figure (2). It is possible to drop any line energy inside the used energy range for calculating the absolute full energy peak efficiency. For example, the sources (U-235 line energy 185.7, U-
238 line energy 1001 and Na-22 line energy 1275 $\mathrm{kev})$, the results were fall directly on the curve as shown in Figure (3), by using all the previous methods (both experimental and MCNP in addition to the fitting equation). However, it can be estimated by using any method from the previous methods (experimental, MCNP in addition to fitting equation).

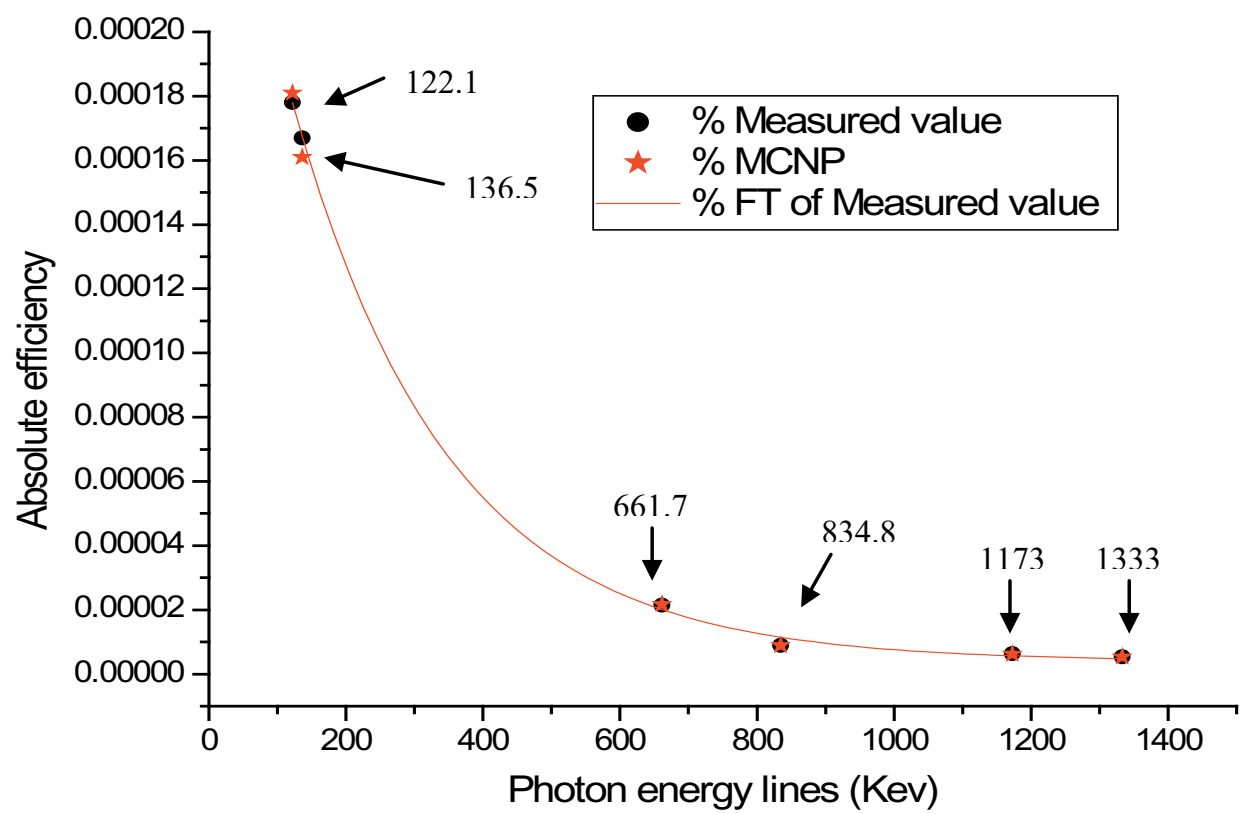

Figure (2). The absolute full energy peak efficiency calculated by MCNP and experimental absolute full energy peak efficiency versus $\gamma$ - energy lines for point sources at D: $40 \mathrm{~cm}$, to extend the curve to cover the high energy range.

[FE has the form: $\left.y=A_{1} e^{*}\left(-x / t_{1}\right)+A_{2} e^{*}\left(-x / t_{2}\right)+A_{3} e^{*}\left(-x / t_{3}\right)+y_{0}\right]$

$\left[y_{0}=0, \quad A_{1}=0.000270504, \quad t_{1}=226.58664, \quad A_{2}=0.0000276331, \quad t_{2}=224.79898, \quad A_{3}=\right.$ $\left.0.00000400308, \quad t_{3}=7.63958 \mathrm{E}+89\right]$

$\mathbf{y}=\varepsilon_{a b}, \quad \mathbf{X}=$ energy line,$\quad \mathbf{F E}=$ Fitting Equation

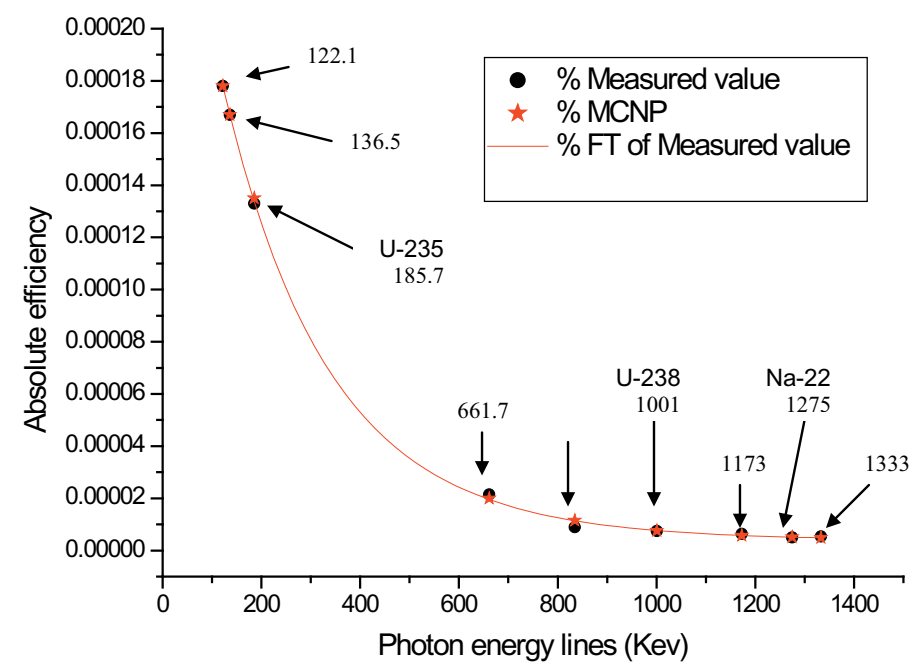

Figure (3). The absolute full energy peak efficiency calculated by MCNP, fitting equation and experimental absolute full energy peak efficiency versus $\gamma$-ray energy lines for point sources at $D$ equal $40 \mathrm{~cm}$. 
Verification of ${ }^{235} \mathrm{U},{ }^{238} \mathrm{U}$ by Mathematical calibration curve

Table (2). Experimental absolute full energy peak efficiency at $D$ equals $40 \mathrm{~cm}$ in comparison with MCNP calculated results for point sources

\begin{tabular}{|c|c|c|c|c|}
\hline \multirow{2}{*}{ Source } & \multirow{2}{*}{$\begin{array}{c}\text { Line } \\
\text { energies } \\
\text { of point } \\
\text { sources } \\
(\mathrm{keV})\end{array}$} & \multicolumn{2}{|c|}{$\begin{array}{c}\varepsilon \\
\pm \boldsymbol{\sigma}_{\xi}\end{array}$} & \multirow{2}{*}{$\begin{array}{c}\text { Accuracy } \\
(\xi \exp -\xi \operatorname{mcnp}) / \\
\xi \exp \%\end{array}$} \\
\hline & & Exp*10-5 & MCNP*10-5 & \\
\hline $\mathrm{Co}-57$ & 122.1 & $17.8 \pm 0.4$ & $18.122 \pm 0.3$ & -1.80899 \\
\hline Cs-137 & 661.7 & $2.145 \pm 0.05$ & $2.17 \pm 0.046$ & -1.1655 \\
\hline $\mathrm{Mn}-54$ & 834.8 & $0.888 \pm 0.015$ & $0.897 \pm 0.012$ & -1.01351 \\
\hline Co-60 & 1173 & $0.63376 \pm 0.011$ & $0.6201 \pm 0.013$ & 2.15539 \\
\hline $\mathrm{Na}-22$ & 1275 & $0.5025 \pm 0.009$ & $0.491 \pm 0.0088$ & 2.288557 \\
\hline Co-60 & 1333 & $0.53444 \pm 0.0098$ & $0.529 \pm 0.0084$ & 1.017888 \\
\hline Co-57 & 136.5 & $16.677 \pm 0.035$ & $16.1 \pm 0.032$ & 3.459855 \\
\hline U-235 & 185.7 & $13.311 \pm 0.022$ & $13.354 \pm 0.021$ & -0.23758 \\
\hline
\end{tabular}

Based on Figure (3) which shows the efficiency using different approaches (experimental, MCNP and fitting equation), it can be used to assay and determine the U-235 at $185.7 \mathrm{kev}$ and $\mathrm{U}-238$ at $1001 \mathrm{kev}$ mass content. These values can be used to determine the enrichment (U-235/ $\mathrm{U}-238$ ) isotopic ratio.

For comparison, the differences between the experimental and calculated results by MCNP are given in Table (2).

The accuracy was found to be between $0.23758 \%$ and $3.459855 \%$, and this indicated that identical values obtained from MCNP and the experimental results and it is more better than the value exhibited in reference [24] which ranged from $3.81 \%$ to $6.02 \%$ From the presented results in Table (2), the following observations can be recorded:

1- Mathematical calibration curve by using
MCNP can be extended to the high $\gamma$-energy.

2- Any energy line for any source can be represented by falling the line on the efficiency curve either by interpolation or extrapolation of the measurable energies.

For comparison, the differences between the fitting equation, experimental and calculated results by MCNP are given in Table (3).

Finally, the following observations can be recorded:-

1- It is notable that the accuracy is better for MCNP with the experimentally measurable values and it is perfect at the beginning of the curve. So, a mathematical calibration curve for the apparatus from MCNP, can be easily calculated.

2- A mathematical calibration curve can be made for the absolute full energy peak efficiency

Table (3). Experimental absolute full energy peak efficiency at $D$ equals $40 \mathrm{~cm}$ in comparison with MCNP calculated and fitting equation results for point sources

\begin{tabular}{|c|c|c|c|c|c|c|c|}
\hline \multirow[b]{2}{*}{ Source } & \multirow{2}{*}{ 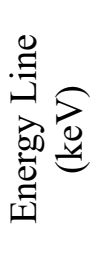 } & \multicolumn{3}{|c|}{$\varepsilon_{\mathrm{ab}}$} & \multicolumn{3}{|c|}{ Accuracy } \\
\hline & & Cal. $* 10^{-5}$ & $\exp ^{*} 10^{-5}$ & menp $* 10^{-5}$ & $\begin{array}{c}\left(\varepsilon_{a b} \text { Cal }\right. \\
-\varepsilon_{a b} \\
\text { menp) } / \\
\varepsilon_{a b} \\
\text { menp \% }\end{array}$ & $\begin{array}{c}\left(\varepsilon_{\mathrm{ab}} \mathrm{Cal}\right. \\
-\varepsilon_{\mathrm{ab}} \\
\exp ) / \varepsilon_{\mathrm{ab}} \\
\exp \%\end{array}$ & $\begin{array}{c}\left(\varepsilon_{\mathrm{ab}} \exp \right. \\
-\varepsilon_{\mathrm{ab}} \\
\mathrm{mcnp}) / \\
\varepsilon_{\mathrm{ab}} \exp \\
\%\end{array}$ \\
\hline U-235 & 185.7 & $13.5 \pm 0.025$ & $13.311 \pm 0.022$ & $13.354 \pm 0.021$ & 1.31 & 3.2 & -0.237 \\
\hline $\mathrm{U}-238$ & 1001 & $0.759 \pm 0.011$ & - & $0.745 \pm 0.016$ & 1.8 & 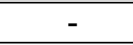 & - \\
\hline $\mathrm{Na}-22$ & 1275 & $0.507 \pm 0.003$ & $0.502 \pm 0.009$ & $0.491 \pm 0.0088$ & 3.3 & 0.832 & 2.2885 \\
\hline Co-60 & 1333 & - & $0.534 \pm 0.0098$ & $0.529 \pm 0.0084$ & 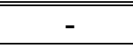 & $-\overline{-}$ & 1.0178 \\
\hline
\end{tabular}


for detector by using MCNP, in order to identify any point sources.

However, a mathematical calibration curve for any detector can be obtained by using MCNP without any experimental measurements, since sources that would be known must have similar specifications of the constructed curve.

\section{Conclusion}

From the obtained results it can be concluded that:

1- It is notable that the fitting is identical for the experimental curve and MCNP calculation, the two methods from the previous methods (experimental and MCNP) can be used to extend the curve to cover the high energy range.

2- It is possible to drop any line energy inside the used energy range for absolute full energy peak efficiency.

3- it can be estimated by using any method from the previous methods (experimental, MCNP and fitting equation).

However, a mathematical calibration curve for any detector can be made by using MCNP without any experimental measurements. So, similar sources must have similar specifications on the constructed curve.

\section{REFERENCES}

[1] IAEA, International Nuclear Verification Series No. 2, Vienna, Austria (1998).

[2] IAEA, IAEA/SG/INF/2, IAEA, Vienna, Austria, (1980).

[3] IAEA, Department of safeguards, STR-327, (2001).

[4] Eurachem, Eurachem working group on uncertainty in chemical measurement, (1995).

[5] R.C. Hagenauer, Nucl. Instr. and Meth. A, 242 (1986) 574

[6] I. Badawy and W.A. El-Gammal, Proceedings of the Sixth National Radio Science Conference H12, Cairo, Egypt, 1999, pp. 1-8.
[7] D. Karamanis, Nucl. Instr. and Meth. A, 505 (1-2) (2003) 282.

[8] I.O.B. Ewa, D. Bodizs, Sz. Czifrus and Zs. Molnar, Appl. Radiat. Isot., 55(1) (2001) 103.

[9] M.C. Le' py, T. Altzitzoglou and D. Arnold, Appl. Radiat. Isot., 55 (2001) 493.

[10] K.M. Wainio and G.F. Knoll, Nucl. Instr. and Meth. A, 44 (2) (1966) 213.

[11] N.V. de Castro and R.J.A. Le' vesque, Nucl. Instr. and Meth. A, 46 (2), (1967) 325.

[12] C. Meixne, Nucl. Instr. and Meth. A 119 (1974) 521.

[13] F.T. Avignone, Nucl. Instr. and Meth. A, 174 (3) (1980) 555.

[14] B.J. Varley, J.E. Kitching, W. Leo, J. Miskin, R.B. Moore, K.D. Wunsch, R. Decker, H. Wollnik and G. Siegert, Nucl. Instr. and Meth. A,190 (3) (1981) 543.

[15] C. Michel, H. Emling, E. Grosse, F. Azgui, H. Grein, H.J. Wollersheim, J.J. Gaardhoje and B. Herskind, Nucl. Instr. and Meth. A, 251 (1) (1986) 119.

[16] S. Hurtado, M. Garci' a-Leo' n and R. Garci' a-Tenorio, Appl. Radiat. Isot., 61 (2-3) (2004) 139.

[17] C.M. Salgado, C.C. Conti and P.H.B. Becker, Appl. Radiat. Isot., 64 (6) (2006) 700.

[18] G. Fehrenbacher, R. Meckbach and P. Jacob, Nucl. Instr. and Meth. A, 383 (1996) 454.

[19] M.A. Ludington and, R.G. Helmer, Nucl. Instr. and Meth. A 446 (3) (2000) 506.

[20] T.F. Wang, J.B. Carlson, Z.M. Koenig, W.D. Ruhter, T.S.H. Lee and J. Winn, Nucl. Instr. and Meth. A, 353 (1-3) (1994) 678.

[21] P.P. Maleka and M. Mauc ${ }^{\sim}$ ec, Nucl. Instr. and Meth. A, 538 (1-3) (2005) 631.

[22] Badawy, A. and El-Gammal, W.A., Symposium on international safeguards: Verification and nuclear material security Vienna (Austria) 29 Oct - 2 Nov 2001, IAEA-SM--367.

[23] USA-National Bureau of Standards, Standard Reference Material 969, Uranium Isotopic Reference Material for gamma spectrometry measurements, NBS-USA (1985); USA-Eckert \& Ziegler, Certified Standard Point Sources, California-USA. (2006).

[24] J.L. Gutie 'rrez-Villanueva, A. Martı 'n-Martı 'n, V.Pe na, M.P. Iniguez and B. de Celis, Journal of Enviromental Radioactivity, 99 (2008) 1520. 